\title{
Toward understanding household preference for consumption characteristics of millet varieties: a case study from western Niger
}

\author{
Jupiter Ndjeunga ${ }^{\mathrm{a}}$, Carl H. Nelson ${ }^{\mathrm{b}, *}$ \\ a ICRISAT, BP 12404, Niamey, Niger \\ ${ }^{\mathrm{b}}$ Department of Agricultural and Consumer Economics, 311 Mumford Hall, 1301 W. Gregory Dr., \\ University of Illinois at Urbana-Champaign, USA
}

Received 6 February 2002; received in revised form 8 May 2003; accepted 5 June 2003

\begin{abstract}
In this study, we evaluate the preference of consumers in Niger for different tuwo or couscous characteristics using a random utility-based choice experiment, ordered probit analysis, and tree-based partitioning. Data were collected through a structured survey administered at four sites. Preferences are estimated for three products (couscous, fermented tuwo, and nonfermented tuwo ) made from five pearl millet cutivars. We provide relative valuation for different traits by type of product. Results show that product taste, color, and textural attributes are important, especially for tuwo and couscous. Probit and partitioning results show that taste and color are the first attributes that consumers use to distinguish more preferred millet food products from less preferred millet food products. This should provide some direction for millet breeding programs and food processing of millet.
\end{abstract}

JEL classification: D1; O1; Q1

Keywords: Pearl millet genetics; Characteristics; Random utility; Ordered probit; Food processing

\section{Introduction}

Pearl millet (Pennisetum glaucum L. Br.) is the primary food source for millions of people in the semiarid tropics of West Africa. In Niger, pearl millet accounted for $77 \%$ of per capita consumption on average between 1994 and 1996 (Nelson and Ndjeunga, 1999). Pearl millet ranks first in terms of total cereal cultivated and production. It represented $72 \%$ of total cereal area and $80 \%$ of total cereal production in Niger for the period 1995-1997. Pearl millet is a subsistence crop, mainly consumed in the form of thick porridges

${ }^{*}$ Corresponding author. Tel.: 217-333-1822.

E-mail address: chnelson@uiuc.edu (C. H. Nelson). (e.g., tuwo ${ }^{1}$ ), thin porridges (e.g., fura, coco, bita), cakes (e.g., massa), or steamed granulated products such as couscous ${ }^{2}$ (ROCAFREMI, 1997).

\footnotetext{
${ }^{1}$ Tuwo is a stiff porridge made from sorghum, pearl millet, maize, rice, or fingermillet flour. It is known in different countries by names such as tuwo in Niger, tô in Burkina Faso and Mali, askfor or eko tutu in Nigeria, ugali in Kenya, atab in Uganda, aceda in Sudan, bogode jiva ting in Botswana, tuo jaafi in Ghana, and sakanti in India (Aboubakar, 1992; Murty and Kumar, 1995). Tuwo is usually eaten with fingers and is accompanied by some types of sauce or stew made of many ingredients. The ingredients used vary among countries and their uses are dependent upon cost and availability. In Niger, the major ingredients used in the preparation of the sauce include tomato, okra, or baobab leaves and meat (Aboubakar, 1992).

${ }^{2}$ Couscous is a steamed granulated product made from sorghum, pearl millet, maize, fonio, or wheat. For immediate use, the product
} 
Breeders have developed more than 17 improved pearl varieties in Niger $^{3}$ (INRAN, 1984). But few of these varieties have been adopted by farmers. Poor adoption of improved varieties is partially explained by seed supply and demand constraints of the available varieties. Seed supply constraints in West Africa have been well researched. They include the low supply of breeder seed, poor seed demand estimation, poor distribution systems, and low seed quality (Ndjeunga, 1997). In contrast, variety-specific demand constraints for millet have not been thoroughly researched. Demand for varieties is a function of plant, grain, and cooking traits preferred by farmers that are embodied in the varieties. The plant and grain traits preferred by farmers have been investigated for pearl millet and groundnut in Niger (Baidu-Forson, 1997; BaiduForson et al., 1997). Sorghum cooking traits have been well researched as well (Aboubakar, 1992; Boling et al., 1982; Da et al., 1982; Gebrekian and GebreHiwot, 1982; Jeannette et al., 1987; Mukuru et al., 1982; Obilana, 1982; Scheuring et al., 1982). However, knowledge of pearl millet cooking traits preferred by consumers is still limited. Farmers' rejection of varieties may be due to their poor cooking traits (Robins, 1995). Therefore, knowledge of traits preferred by consumers is valuable for crop improvement programs and provides market signals for food processors. The demand for improved pearl millet varieties is likely to increase if, among others, varieties are designed to include producers' and consumers' preferred cooking traits. Improving the performance of varieties in terms of cooking quality traits will contribute to the productivity, efficiency, and profitability of pearl millet production in Niger.

This study systematically evaluates the preferences of consumers for tuwo and couscous cooking quality characteristics. It uses ordered probit analysis and tree-based partitioning to measure the value of tuwo and couscous cooking quality characteristics. Random utility-based choice experimentation is a surveybased valuation technique, widely used in marketing

is sprinkled with water and mixed thoroughly after adding baobab (Adansonia digitata L.) leaf powder or okra powder. This flour aggregate mixture is again steamed for about 25 minutes to give the couscous product a light texture.(Murty and Kumar, 1995).

${ }^{3}$ A large number of other improved pearl millet varieties are awaiting release in Niger. They are listed as: ICMV89305, ICMV 92222, ICMV 94206, GB 8735, MTDO92, MTTY92, and CTO-V. research, which relies on individuals' background and judgment of products to estimate the marginal contribution of specific product traits to overall preferences. Random utility-based preference elicitation provides an analysis of preference that is consistent with actual choice, and is founded in a behavioral theory (Louviere, 2001). Consumer's relative preferences for attributes are estimated with ordered probit models. Tree-based partitioning is a rigorous statistical classification procedure that supplements the results of ordered probit models by producing information about the order in which attributes lead to preference distinctions.

\section{Empirical design and data collection}

The development of a survey instrument to generate data that would allow the analysis of the independent effects of mutually exclusive consumption attributes involved a number of steps. First, available literature on prior economic and food science analyses of consumer preference for attributes was reviewed to develop a list of important tuwo and couscous characteristics for potential inclusion in the questionnaire. Texture, color, taste, and keeping quality are often cited as tuwo quality characteristics that are important for sorghum consumers (Aboubakar, 1992; Boling and Eisener, 1982; Da et al., 1982; Fliedel et al., 1998; Gebrekian and GebreHiwot, 1982; Mukuru et al., 1982; Murty et al., 1982; Obilana, 1982; Scheuring et al., 1982). Few sensory studies have been documented on pearl millet quality. A study undertaken on couscous quality characteristics in three villages in Niger indicated that color, taste, and texture were the most important quality traits (Oumarou et al., 1998). These characteristics, likely to be preferred by consumers, were used to conduct a focus group meeting with pearl millet consumers from two villages: Berikoira and Tagabati. The meetings were conducted to evaluate alternative question formats and contents, and to elicit general advice about mutually exclusive traits desirable to consumers. The information from the focus group meetings was used to classify consumption attributes into unique mutually exclusive categories (Louviere, 1990, p. 51). The last stage was the development of the survey instrument.

The characteristics included in the survey instrument were those ranked high in the focus group meetings. 
In general, a couscous of light color (white cream or yellow), with a nice aroma and taste, and with a good overall texture is likely to be most preferred. Texture is a composite of cohesiveness, stickiness, and chewiness. A couscous that does not feel hard to the touch, is not sticky, and is not hard to chew would have good texture. A tuwo with light color, good taste, good texture, and good overnight keeping quality is likely to be preferred by consumers. Taste has been found to be strongly influenced by color and texture. The desirable textural attributes are hardness, cohesiveness, and adhesiveness. Because tuwo is eaten with fingers it should be firm enough to scoop a piece that holds together and does not crumble under finger pressure. Consumers do not want tuwo to stick to the fingers, teeth, or palate. Tuwo left over from a meal is often stored overnight, and consumed the following day. Thus, a good tuwo should remain stiff during overnight storage.

The survey included 7 couscous characteristics and 11 tuwo characteristics. Couscous products were evaluated on the basis of: color (disliked, liked); aroma (disliked, liked); cohesiveness (soft, just right, hard); stickiness (not sticky, just right, too sticky); taste (disliked, liked); chewiness (disliked, liked); and texture (disliked, liked). Tuwo products were evaluated on the basis of: color (disliked, liked); appearance-interior (disliked, liked); aroma (disliked, liked); cohesiveness (soft, just right, hard); stickiness (not sticky, just right, too sticky); consistency (disliked, liked); taste (disliked, liked); the way the tuwo dissolves in the mouth (slow, just right, fast); chewiness (disliked, liked); texture (disliked, liked); and tuwo overnight keeping quality (not consistent, just right, too consistent). The aggregate attribute texture was included as a preference consistency check. Respondents evaluated couscous and tuwo made from five different varieties, ranking the varieties on a 5-point preference scale ( 1 being the least preferred and 5 being the most preferred). Tuwo and couscous products were processed from five pearl millet varieties: four improved varieties, MTDO92, Zatib, Souna III, and CTO-V, and a local cultivar, Hainikiré. These cultivars were recommended by pearl millet breeders and food technologists and varied in pericarp color and endosperm texture. The improved cultivars were grown in the Dosso department in Niger during the 1998 crop season. Traditional technology was used to process all products. In each site and for each variety, a local woman was hired to decorticate the grain, mill the decorticated grain into fine flour, and process the flour into tuwo or couscous. ${ }^{4}$

The panellists used in this experiment were a mixture of literate and illiterate adults, averaging 44 years of age. A total of 114 panellists were selected on the basis of ethnicity, gender, and urbanization. About half the consumers were adult female. Two-thirds of the panel came from the Zarma ethnic group and about one third from the Haoussa ethnic group. About one third of the panellists were literate. More than $80 \%$ of the zarma ethnic group panellists and $50 \%$ of the the haoussa ethnic group panellists ate tuwo or couscous at least once a day, respectively. Table 1 presents a summary of the characteristics of the survey sample gender, age, household size, ethnic group, level of education, and daily consumption frequency of tuwo or couscous.

During the first two days of the survey, enumerators collected information on the respondents' socioeconomic profile. During the third day, panellists familiarized themselves with the terminology used and the ranking scales. Tests were carried out at ambient temperature in three separate rooms with one enumerator and a food technologist per room. Panellists were asked to avoid communication during the test. On each of four successive days, the panel evaluated five nonfermented tuwo samples made from the five pearl millet varieties. The next day, panellists were asked to evaluate the overnight keeping quality of the tuwo. The same day, respondents also evaluated five couscous products made from the same five pearl millet varieties. During the last two days, the panellists were asked to evaluate five fermented tuwo products and their keeping qualities.

The local variety control for all products was the pearl millet variety used in the village. In each case, the local cultivar Hainikiré was used. Each of the panel members evaluated the product using the cards presented in Table 2 for tuwo and in Table 3 for couscous. The questionnaire covered all relevant attributes of tuwo and couscous. The tables indicate that different attributes were evaluated at different stages of food preparation and consumption. For example, the cohesiveness of tuwo is evaluated when a piece of tuwo is cut, and the stickiness is evaluated after the piece

\footnotetext{
${ }^{4}$ Details about the preparation of tuwo and couscous can be obtained from the authors on request.
} 
Table 1

Characteristics of consumer surveyed in Niger

\begin{tabular}{|c|c|c|c|c|c|}
\hline Survey site & Berikoira & Karabedji & Gaweye & Chikal & Total \\
\hline Sample size & 30 & 30 & 25 & 29 & 114 \\
\hline \multicolumn{6}{|l|}{ 1. Distribution of respondents by gender } \\
\hline Men & 15 & 15 & 13 & 15 & 58 \\
\hline Women & 15 & 15 & 12 & 14 & 56 \\
\hline \multicolumn{6}{|l|}{ 2. Age distribution in years } \\
\hline Average (years) & 45 & 41 & 45 & 46 & 44 \\
\hline$\leq 30$ years & 3 & 4 & 2 & 3 & 12 \\
\hline 30 years & 27 & 26 & 23 & 26 & 102 \\
\hline 3. Average household size & 13 & 9 & 6 & 11 & 10 \\
\hline Less than 4 members per household & 0 & 1 & 6 & 1 & 8 \\
\hline Between 4 and 8 members & 8 & 15 & 16 & 12 & 51 \\
\hline More than 8 members & 22 & 14 & 3 & 16 & 55 \\
\hline \multicolumn{6}{|l|}{ 4. Ethnic groups } \\
\hline Zarma & 30 & 30 & 18 & 0 & 78 \\
\hline Haoussa & 0 & 0 & 1 & 29 & 30 \\
\hline Others (Gourma, Gaboro, Peulh, Dendi) & 0 & 0 & 6 & 0 & 6 \\
\hline \multicolumn{6}{|l|}{ 5. Level of education } \\
\hline Illiterate & 21 & 25 & 13 & 24 & 83 \\
\hline Literate $^{\mathrm{a}}$ & 9 & 5 & 12 & 5 & 31 \\
\hline \multirow{2}{*}{\multicolumn{6}{|c|}{$\begin{array}{l}\text { 6. Daily consumption frequency for } \\
\text { Tuwo }\end{array}$}} \\
\hline & & & & & \\
\hline Less than 1 tuwo per day & 0 & 0 & 5 & 15 & 20 \\
\hline More than 1 tuwo per day & 30 & 30 & 20 & 14 & 94 \\
\hline \multicolumn{6}{|l|}{ Couscous } \\
\hline Less than 1 couscous per day & 14 & 12 & 12 & 21 & 59 \\
\hline More than 1 couscous per day & 16 & 18 & 13 & 8 & 55 \\
\hline
\end{tabular}

${ }^{\mathrm{a}}$ Includes all panellists with koranic, primary, or secondary education.

of tuwo has been worked up in the hand. Due to time constraints faced by consumers in urban Niamey, the fermented tuwo test could not be implemented. Thus, results reported for fermented tuwo cover three sites: Berikoira, Karabedji, and Chikal.

\section{Theoretical framework}

The survey data were analyzed with a random utility behavioral model of consumption that explains variety choice by preferences for product characteristics, as in the Lancasterian theory of consumer choice (Hamath et al., 1997). This characteristic-based choice model was used to evaluate preference for tuwo and couscous characteristics. In this model, the characteristics of the goods generate utility for consumers (Lancaster, 1991). The specification of intrinsic properties of goods as arguments of the utility function, and the possibility of confining analysis to goods that yield common characteristics are important in empirical demand analysis (Ratchford 1975).

Let $U$ represent utility for an individual. Utility is hypothesized to be a function of various factors, including the characteristics of the products, $S$, the individuals' socioeconomic background, $Z$, and an interaction term between the individuals' background and the products' characteristics, $\mu$. The decision maker will choose the product that provides the highest utility (Train, 1986). That is, the decision maker will choose product $j$ over $j^{\prime}$ if and only if $U_{j}>U_{j}^{\prime}$. The utility function can be formally written as

$$
\begin{gathered}
U_{j}=f\left(S_{1 j}, S_{2 j}, \ldots, S_{g j} ; Z_{1}, Z_{2}, \ldots, Z_{i} ;\right. \\
\left.\mu_{1}, \mu_{2}, \ldots, \mu_{g j i} \mid \Theta_{g}\right)+e
\end{gathered}
$$

where $j=1,2, \ldots, m ; g=1,2, \ldots, k ; i=1,2, \ldots, n$. Here $j$ represents the product from a given variety; $g$ the preference traits for the product, and $i$ each consumer's socioeconomic characteristics. The variables $s$ and $Z$ 
Table 2

Typical card for tuwo from the survey

\begin{tabular}{|c|c|}
\hline Variables & $\begin{array}{l}\text { Product } i \\
(i=1,2, \ldots, 5)\end{array}$ \\
\hline \multicolumn{2}{|l|}{ Look at the tuwo, don't touch or cut it. } \\
\hline \multicolumn{2}{|l|}{ Cut of piece of tuwo, but don't eat it. } \\
\hline Do you like the way the interior looks? & Liked/disliked \\
\hline Do you like the aroma? & Liked/disliked \\
\hline How hard is the tuwo? & Soft/just right/hard \\
\hline \multicolumn{2}{|l|}{$\begin{array}{l}\text { Take a piece of tuwo and work up the piece } \\
\text { with your hands. }\end{array}$} \\
\hline $\begin{array}{l}\text { How do you like the way the tuwo holds } \\
\text { together? }\end{array}$ & Liked/disliked \\
\hline How sticky is the tuwo to your fingers? & $\begin{array}{c}\text { Not sticky/just } \\
\text { right/sticky }\end{array}$ \\
\hline \multicolumn{2}{|l|}{ Now, eat as much tuwo as you want and tell me: } \\
\hline How do you like the taste? & Liked/disliked \\
\hline How do you like the chewiness? & Liked/disliked \\
\hline How quickly does it dissolve in your mouth? & Slow/just right/fast \\
\hline How do you like the overall texture? & Liked/disliked \\
\hline How do you like its consistency after a night? & Liked/disliked \\
\hline Rating of the products tested? & \\
\hline
\end{tabular}

are the main effect variables representing the products' attributes and the individuals' profile, respectively. The term $\mu_{g j i}=S_{g j} \times Z_{i}$, is the interaction variable between the products' characteristics and individuals' profiles. Since only a portion of the arguments in the above equation is observed, the equation is stochastic and variable $e$ is a spherical disturbance term. The parameter estimates are represented by the vector $\Theta_{g}$.

Table 3

Typical card for couscous from the survey

\begin{tabular}{ll}
\hline Variables & $\begin{array}{l}\text { Product } i \\
(i=1,2, \ldots, 5)\end{array}$ \\
\hline $\begin{array}{l}\text { Look at the couscous, don't touch it. } \\
\text { How much do you like the color? }\end{array}$ & Liked/disliked \\
$\begin{array}{l}\text { Take a piece of couscous, but don't eat it. } \\
\text { How much do you like the aroma? }\end{array}$ & Liked/disliked \\
How hard is the couscous? & Soft/just right/hard \\
Take a piece of couscous and work up the piece. & Not sticky/just \\
How sticky is the couscous to & right/sticky \\
$\quad$ your fingers? & \\
$\begin{array}{l}\text { Now, eat as much couscous } \text { as you want } \\
\text { and answer the following questions. }\end{array}$ & Liked/disliked \\
How much do you like the taste? & Liked/disliked \\
How much do you like the chewiness? & Liked/disliked \\
How much do you like the overall texture? & \\
Rating of the products tested? &
\end{tabular}

Market researchers have used different approaches, compositional and decompositional, to estimate the vector $\Theta_{g}$. The decompositional approach uses measures of preference (e.g, a ranking) for multivariate alternatives or products to estimate the values attached to underlying characteristics. The partial derivative of the jth product (e.g., tuwo) with respect to the products' characteristics (e.g., color), gives the value of the partworth that the consumer assigns to the $g$ th characteristic level of the $j$ th product (Louviere, 1990). Since the utility of a product to an individual is a function of both the product's characteristics and the individuals' profile, the partworth is a joint effect of the two variables (Moore, 1980). For example, the change of utility of a tuwo to a consumer is determined by the variation of the tuwo characteristics and background of the consumer. More formally,

$\frac{\partial U\left(s^{*}\right)}{\partial S_{g}}=\frac{\partial f(\cdot)}{\partial S_{g}}+\frac{\partial f(\cdot)}{\partial \mu_{g}} \times \frac{\partial \mu_{g}}{\partial S_{g}}$

where the first side of the equation is the partworth of the gth level of the characteristic of a product to a given individual. It is composed of two parts. The first, the marginal value of the $g$ th attribute level, measures the changes in the utility when only product attributes levels are allowed to vary and will be referred to as $V_{g}$. The second part is a product of two partials. The first term measures the variation in the utility associated with changes in the interaction term. It is also referred as the weight factor $\left(b_{g}\right)$. The second term of the combination, $Z_{i}$, represents an individual's socioeconomic background. In general, the partworth of a product's attribute to an individual can be represented easily by

$$
\frac{\partial U\left(s^{*}\right)}{\partial S_{g}}=V_{g}+Z_{i} \times b_{g} .
$$

The weight factor, $b$, is hypothesized to take any value from negative infinity to positive infinity and captures the variability in preferences for a product due to the interaction between a person's background and product attributes. This is a direct measure of segmentability of the market. People with the same $b$ coefficient have similar preferences, and hence can be grouped into one segment. Partially differentiating equation (1) with respect to individuals' profiles, gives variations in the utility that are accounted for by variation in socioeconomic variables across individuals. In 
many cases, one is not interested at all in the main effects due to personal attributes, since the main effects may only reflect response biases (Green and DeSarbo, 1979). The relative importance of products for respondents can be computed using estimates from equation (1). For example, how important is one tuwo attribute relative to all other tuwo characteristics? The formula for the relative importance is

$\psi_{a}=\frac{\left[\max \left(v_{g a}^{*}\right)-\min \left(v_{g a}^{*}\right)\right]}{\sum \omega_{a}}$

where $v_{g a}^{*}$ is the marginal value of the $g$ th level of the $a$ th attribute, $\psi_{a}$ represents the relative importance for the $a$ th attribute, $\sum \omega_{a}$ is the sum of all ranges $\left[\max \left(v_{g a}^{*}\right)-\min \left(v_{g a}^{*}\right)\right]$, across all attributes. $\psi_{a}$ for a consumer can be normalized to ascertain its relative importance with regard to the other attributes and across consumers (Jain et al., 1979).

\section{Estimation technique and the econometric model}

OLS estimation is sometimes employed to analyze choice experiments, despite its limitations in analyzing data with categorical dependent variables ${ }^{5}$ (Doyle, 1977). The linear probability model is a procedure for discrete dependent variables, but it has a number of shortcomings. The error term is heteroskedastic, so it produces inefficient estimates (Greene, 1990, p. 663). Because of these difficulties, an ordered probit model will be specified and estimated. The specified model consists of $U$ as an unobservable dependent variable, $R_{r}$ (where $\left.\gamma=0,1,2,3, \ldots, w\right)$ as the choice alternative or observable dependent variable, $v$ and $\mu$ as the block of independent variables, and $\gamma$ as the threshold variable. It can be formulated as

$U=\alpha+S v+\mu b+e$

where $e \sim N(0,1)$ and $R=0$ if $U \leq 0, R=1$ if $U \leq$ $\gamma_{1}, R=2$ if $\gamma_{1} \leq U \leq \gamma_{2}, \ldots$, and $R=w$ if $\gamma_{\mathrm{w}-2} \leq U . U$ is a $j \times 1$ vector of unobservable utility, of, say, a tuwo from a pearl millet cultivar, and $R_{\gamma}$ is

\footnotetext{
${ }^{5}$ Maddala refers to the preference measured on a scale of $1,2, \ldots$, 5 with 1 being intensely disliked and 5 being intensely liked as an ordered categorical variable (Maddala, 1993).
}

a vector of preference rankings. The $\gamma$ 's are threshold variables or cut-off points that provide the ratings of alternatives, $S$ is a matrix of nonstochastic effect-coded variables of $N$ attribute levels for the $M$ products; $v$ is a matrix of marginal values of the $g$ th characteristic level for the $j$ th product; and $\mu$ is another matrix of nonstochastic interaction variables of $M$ products and $N$ individuals' characteristics. The interaction terms are effect coded $(-1,0,1) ; b$ is the weight of the interaction term between the $g$ th product's attribute and the $i$ th consumer's characteristics; $\alpha$ is a column vector of constants or the intercept of the equation; and $e$ is the stochastic error term.

The threshold concept is central to the economic theory of consumer behavior (Doyle, 1977). The theory asserts that a buyer responds (buys or rates alternatives) when utility exceeds a threshold or critical level of satisfaction. For example, variations in the independent variable would cause a switch in a consumer's preference ranking when utility reaches some levels. The cut-off points vary with individuals. Individuals with similar tastes and background have similar cutoff points.

The ordered probit analysis of the choice experiment data was supplemented by a classification tree analysis (Breiman et al., 1984). Categorical classification trees are statistical models used to classify categorical data or uncover its predictive structure. An example is predicting a patient's risk for a particular disease based on observed characteristics of the patient. In this application, we are interested in finding the attributes that lead to the strongest preference for millet varieties.

The procedure rpart implemented in S-PLUS (Venables and Ripley, 1999) was used to estimate the classification tree. This tree-based estimation procedure makes one-step ahead classification splits to minimize the sum of squares from the split. The choice is verified by running a 10 -fold cross-validation (Venables and Ripley, 1999, p. 310).

\section{Results and discussions}

\subsection{Ranking and attribute levels}

Tables 4-6 present two-way contingency tables of the various sensory attributes and Pearson chisquared test statistics of the hypothesis of statistical 
Table 4

Comparison of the 5 pearl millet cultivars for nonfermented tuwo sensory attributes ratings

\begin{tabular}{|c|c|c|c|c|c|c|c|c|c|}
\hline Attribute & Value & Hainikiré & MTDO92 & ZATIB & Souna III & CTO-V & Pearson $\chi^{2}$ & Degrees of freedom & $P$-value \\
\hline \multirow[t]{2}{*}{ Color } & 0 & 6 & 11 & 60 & 40 & 50 & 96.52 & 4 & 0.000 \\
\hline & 1 & 108 & 103 & 54 & 74 & 64 & & & \\
\hline \multirow[t]{2}{*}{ Appearance (interior) } & 0 & 9 & 18 & 45 & 28 & 43 & 45.42 & 4 & 0.000 \\
\hline & 1 & 105 & 96 & 69 & 86 & 71 & & & \\
\hline \multirow[t]{2}{*}{ Aroma } & 0 & 9 & 22 & 53 & 28 & 35 & 48.64 & 4 & 0.000 \\
\hline & 1 & 105 & 92 & 61 & 86 & 79 & & & \\
\hline \multirow[t]{3}{*}{ Cohesiveness } & 0 & 31 & 36 & 11 & 17 & 33 & 69.67 & 8 & 0.000 \\
\hline & 1 & 63 & 55 & 35 & 49 & 51 & & & \\
\hline & 2 & 20 & 23 & 68 & 48 & 30 & & & \\
\hline \multirow[t]{2}{*}{ Firmness } & 0 & 12 & 28 & 41 & 26 & 42 & 27.66 & 4 & 0.000 \\
\hline & 1 & 102 & 86 & 73 & 88 & 72 & & & \\
\hline \multirow[t]{3}{*}{ Stickiness } & 0 & 39 & 27 & 38 & 43 & 22 & 16.60 & 8 & 0.035 \\
\hline & 1 & 43 & 49 & 34 & 41 & 50 & & & \\
\hline & 2 & 32 & 38 & 42 & 30 & 42 & & & \\
\hline \multirow[t]{2}{*}{ Taste } & 0 & 17 & 19 & 36 & 22 & 34 & 15.58 & 4 & 0.004 \\
\hline & 1 & 97 & 95 & 78 & 92 & 80 & & & \\
\hline \multirow[t]{2}{*}{ Chewiness } & 0 & 12 & 18 & 34 & 22 & 34 & 20.27 & 4 & 0.000 \\
\hline & 1 & 102 & 96 & 80 & 92 & 80 & & & \\
\hline \multirow[t]{3}{*}{ Mouth feel } & 0 & 37 & 44 & 61 & 44 & 49 & 26.55 & 8 & 0.001 \\
\hline & 1 & 32 & 23 & 34 & 39 & 26 & & & \\
\hline & 2 & 45 & 47 & 19 & 31 & 39 & & & \\
\hline \multirow[t]{2}{*}{ Texture } & 0 & 11 & 19 & 30 & 20 & 35 & 19.72 & 4 & 0.001 \\
\hline & 1 & 103 & 95 & 84 & 94 & 79 & & & \\
\hline \multirow[t]{12}{*}{ Rank $(\%)$} & 1 & 11 & 15 & 40 & 4 & 44 & 179.21 & 16 & 0.000 \\
\hline & & $(9.7)$ & (13.2) & $(35.1)$ & $(3.5)$ & $(38.6)$ & & & \\
\hline & 2 & 9 & 14 & 26 & 46 & 19 & & & \\
\hline & & (7.9) & (12.3) & $(22.8)$ & $(40.4)$ & (16.7) & & & \\
\hline & 3 & 15 & 14 & 21 & 34 & 30 & & & \\
\hline & & (13.2) & (12.3) & (18.4) & $(29.8)$ & $(26.3)$ & & & \\
\hline & 4 & 38 & 36 & 21 & 12 & 7 & & & \\
\hline & & (33.3) & (31.6) & (18.4) & (10.5) & $(6.1)$ & & & \\
\hline & 5 & 41 & 35 & 6 & 18 & 14 & & & \\
\hline & & $(36.0)$ & (30.7) & $(5.3)$ & (15.8) & (12.3) & & & \\
\hline & Total & 114 & 114 & 114 & 114 & 114 & & & \\
\hline & & $(100.0)$ & (100.0) & $(100.0)$ & $(100.0)$ & $(100.0)$ & & & \\
\hline
\end{tabular}

Color: $(0=$ dislike, $1=$ liked $)$ Appearance: $(0=$ dislike, $1=$ liked $)$; Aroma: $(0=$ dislike, $1=$ liked $)$ Cohesiveness: $(0=$ soft, $1=$ average, $2=$ hard $)$; Firmness: $(0=$ dislike, $1=$ liked); Stickiness: $(0=$ not sticky, $1=$ average, $2=$ sticky $)$; Taste: $(0=$ dislike, $1=$ liked $)$; Chewiness: $(0=$ dislike, $1=$ liked $)$; Mouth feel: $(0=$ slow, $1=$ just right, $2=$ fast $)$; Texture: $(0=$ dislike, $1=$ liked $)$.

independence (Agresti, 1990) for nonfermented tuwo, fermented tuwo, and couscous, respectively. The results indicate that the panellists were able to discriminate between samples of the same product for each of the attributes and for their overall acceptability. For nonfermented tuwo, all attributes are perceived to be significantly different across varieties according to the Pearson chi-squared statistic, with Hainikiré showing the highest frequency of preferred attributes and Zatib the lowest (Table 4). Hainikiré and MTDO92 had the best overall rankings, while Zatib had the lowest. Similar patterns were found for fermented tuwo and couscous in Tables 5 and 6 , respectively. The only attribute preference that is not significantly different across varieties is the mouth feel of fermented tuwo. For all three products Hainikiré and MTD092 received the highest ranking and Zatib received the lowest. The Pearson statistics show that all of the attributes other than the mouth feel of fermented tuwo are strongly distinguished. This is evidence that the survey instrument 
Table 5

Comparison of the 5 pearl millet cultivars for fermented tuwo sensory attributes ratings

\begin{tabular}{|c|c|c|c|c|c|c|c|c|c|}
\hline Attribute & Value & Hainikiré & MTDO92 & ZATIB & Souna III & CTO-V & Pearson $\chi^{2}$ & Degrees of freedom & $P$-value \\
\hline \multirow[t]{2}{*}{ Color } & 0 & 4 & 9 & 60 & 26 & 34 & 106.77 & 4 & 0.000 \\
\hline & 1 & 85 & 80 & 29 & 63 & 55 & & & \\
\hline \multirow[t]{2}{*}{ Appearance (interior) } & 0 & 1 & 8 & 43 & 24 & 20 & 69.52 & 4 & 0.000 \\
\hline & 1 & 88 & 81 & 46 & 65 & 69 & & & \\
\hline \multirow[t]{2}{*}{ Aroma } & 0 & 3 & 8 & 47 & 25 & 20 & 74.86 & 4 & 0.000 \\
\hline & 1 & 86 & 81 & 42 & 64 & 69 & & & \\
\hline \multirow[t]{3}{*}{ Cohesiveness } & 0 & 12 & 17 & 33 & 11 & 17 & 33.21 & 8 & 0.000 \\
\hline & 1 & 55 & 38 & 26 & 41 & 36 & & & \\
\hline & 2 & 22 & 34 & 30 & 37 & 36 & & & \\
\hline \multirow[t]{2}{*}{ Firmness } & 0 & 3 & 15 & 37 & 27 & 25 & 41.05 & 4 & 0.000 \\
\hline & 1 & 86 & 74 & 52 & 62 & 64 & & & \\
\hline \multirow[t]{3}{*}{ Stickiness } & 0 & 31 & 24 & 20 & 34 & 26 & 22.34 & 8 & 0.0004 \\
\hline & 1 & 37 & 33 & 22 & 32 & 33 & & & \\
\hline & 2 & 21 & 32 & 47 & 23 & 30 & & & \\
\hline \multirow[t]{2}{*}{ Taste } & 0 & 5 & 9 & 41 & 20 & 20 & 52.33 & 4 & 0.000 \\
\hline & 1 & 84 & 80 & 48 & 69 & 69 & & & \\
\hline Chewiness & 0 & 6 & 13 & 42 & 19 & 24 & 46.60 & 4 & 0.000 \\
\hline \multirow{3}{*}{ Mouth feel } & 0 & 32 & 33 & 49 & 37 & 38 & 10.45 & 8 & 0.235 \\
\hline & 1 & 30 & 23 & 18 & 22 & 24 & & & \\
\hline & 2 & 27 & 33 & 22 & 30 & 27 & & & \\
\hline \multirow[t]{2}{*}{ Texture } & 0 & 8 & 9 & 41 & 21 & 23 & 45.48 & 4 & 0.000 \\
\hline & 1 & 81 & 80 & 48 & 68 & 66 & & & \\
\hline \multirow[t]{10}{*}{ Rank (\%) } & 1 & $\begin{array}{c}4 \\
(45)\end{array}$ & $\begin{array}{l}5 \\
(56)\end{array}$ & $\begin{array}{l}45 \\
(506)\end{array}$ & $\begin{array}{l}13 \\
(146)\end{array}$ & $\begin{array}{l}22 \\
(247)\end{array}$ & 228.43 & 16 & 0.000 \\
\hline & 2 & 3 & 7 & 17 & 38 & 24 & & & \\
\hline & & (3.4) & (7.9) & (19.1) & $(42.7)$ & $(27.0)$ & & & \\
\hline & 3 & $\begin{array}{l}12 \\
(13.5)\end{array}$ & $\begin{array}{l}10 \\
(11.2)\end{array}$ & $\begin{array}{l}20 \\
(22.5)\end{array}$ & $\begin{array}{l}23 \\
(25.8)\end{array}$ & $\begin{array}{l}24 \\
(27.0)\end{array}$ & & & \\
\hline & 4 & 28 & 33 & 5 & 10 & 13 & & & \\
\hline & & $(31.5)$ & $(37.1)$ & (5.6) & (11.2) & (14.6) & & & \\
\hline & 5 & 42 & 34 & 2 & 5 & 6 & & & \\
\hline & & $(47.2)$ & $(38.2)$ & $(2.23)$ & (5.6) & (6.7) & & & \\
\hline & Total & 89 & 89 & 89 & 89 & 89 & & & \\
\hline & & $(100.0)$ & (100.0) & (100.0) & $(100.0)$ & (100.0) & & & \\
\hline
\end{tabular}

Color: $(0=$ dislike, $1=$ liked); Appearance: $(0=$ dislike, $1=$ liked); Aroma: $(0=$ dislike, $1=$ liked $)$; Cohesiveness: $(0=$ soft, $1=$ average, $2=$ hard $)$; Firmness: $(0=$ dislike, $1=$ liked $)$; Stickiness: $(0=$ not sticky, $1=$ average, $2=$ sticky $)$; Taste: $(0=$ dislike, $1=$ liked $)$; Chewiness: $(0=$ dislike, $1=$ liked $)$; Mouth feel: $(0=$ slow, $1=$ just right, $2=$ fast $)$; Texture: $(0=$ dislike, $1=$ liked $)$.

did identify sensory attributes that the members of the households were able to distinguish. These results are consistent with findings on sorghum tuwo quality evaluation in Niger in which appearance and color showed the most discrimination (Aboubakar, 1992).

One-way ANOVA was used to assess differences in traits' ratings based on socioeconomic variables such as gender, ethnic group, level of education, age groups, household size, and daily consumption frequency of tuwo or couscous. The results indicated that consumers' ratings of all traits did not differ by gender, level of education, age, household size, or daily consumption frequency of tuwo or couscous. However, consumers' rating of traits (except for color) significantly differed by ethnic group. Therefore, further analysis included ethnic group as the socioeconomic variable. This was implemented by introducing interactions between attributes and a dummy variable with a value of 0 for the Zarma ethnic group and a value of 1 for the Haoussa ethnic group. 
Table 6

Comparison of the 5 pearl millet cultivars for couscous sensory attributes rating

\begin{tabular}{|c|c|c|c|c|c|c|c|c|c|}
\hline Attribute & Value & Hainikiré & MTDO92 & ZATIB & Souna III & CTO-V & Pearson $\chi^{2}$ & Degrees of freedom & $P$-value \\
\hline \multirow[t]{2}{*}{ Color } & 0 & 5 & 3 & 53 & 47 & 22 & \multirow[t]{2}{*}{107.42} & \multirow[t]{2}{*}{4} & \multirow[t]{2}{*}{0.000} \\
\hline & 1 & 109 & 111 & 61 & 67 & 92 & & & \\
\hline \multirow[t]{2}{*}{ Aroma } & 0 & 12 & 12 & 49 & 42 & 24 & \multirow[t]{2}{*}{55.41} & \multirow[t]{2}{*}{4} & \multirow[t]{2}{*}{0.000} \\
\hline & 1 & 102 & 102 & 65 & 72 & 90 & & & \\
\hline \multirow[t]{3}{*}{ Cohesiveness } & 0 & 33 & 74 & 35 & 23 & 20 & \multirow[t]{3}{*}{103.33} & \multirow[t]{3}{*}{8} & \multirow[t]{3}{*}{0.000} \\
\hline & 1 & 66 & 30 & 46 & 42 & 53 & & & \\
\hline & 2 & 15 & 10 & 33 & 49 & 41 & & & \\
\hline \multirow[t]{3}{*}{ Stickiness } & 0 & 64 & 30 & 44 & 43 & 54 & \multirow[t]{3}{*}{45.23} & \multirow[t]{3}{*}{8} & \multirow[t]{3}{*}{0.000} \\
\hline & 1 & 40 & 41 & 47 & 52 & 45 & & & \\
\hline & 2 & 10 & 43 & 23 & 19 & 15 & & & \\
\hline \multirow[t]{2}{*}{ Taste } & 0 & 12 & 5 & 44 & 39 & 21 & \multirow[t]{2}{*}{57.42} & \multirow[t]{2}{*}{4} & \multirow{2}{*}{0.000} \\
\hline & 1 & 102 & 108 & 70 & 75 & 93 & & & \\
\hline \multirow[t]{2}{*}{ Chewiness } & 0 & 12 & 5 & 41 & 43 & 24 & \multirow[t]{2}{*}{58.92} & \multirow[t]{2}{*}{4} & \multirow[t]{2}{*}{0.000} \\
\hline & 1 & 102 & 109 & 73 & 71 & 90 & & & \\
\hline \multirow[t]{2}{*}{ Texture } & 0 & 16 & 9 & 35 & 32 & 26 & \multirow[t]{2}{*}{25.50} & \multirow[t]{2}{*}{4} & \multirow[t]{2}{*}{0.000} \\
\hline & 1 & 98 & 105 & 79 & 82 & 88 & & & \\
\hline \multirow[t]{11}{*}{ Rank } & 1 & $\begin{array}{l}5 \\
(4.4)\end{array}$ & $\begin{array}{l}11 \\
(9.7)\end{array}$ & $\begin{array}{l}44 \\
(38.6)\end{array}$ & $\begin{array}{c}34 \\
(29.9)\end{array}$ & $\begin{array}{l}20 \\
(17.5)\end{array}$ & \multirow[t]{11}{*}{151.84} & 16 & 0.000 \\
\hline & 2 & 11 & 9 & 26 & 35 & 33 & & & \\
\hline & & $(9.7)$ & (7.9) & (22.8) & (30.7) & (29.0) & & & \\
\hline & 3 & 24 & 15 & 24 & 21 & 30 & & & \\
\hline & & (21.1) & (13.2) & (21.1) & (18.4) & (26.3) & & & \\
\hline & 4 & 41 & 41 & 11 & 8 & 13 & & & \\
\hline & & $(36.0)$ & $(36.0)$ & $(9.7)$ & $(7.0)$ & (11.4) & & & \\
\hline & 5 & 33 & 38 & 9 & 16 & 18 & & & \\
\hline & & (29.0) & (33.3) & $(7.9)$ & (14.0) & (15.8) & & & \\
\hline & Total & 114 & 114 & 114 & 114 & 114 & & & \\
\hline & & $(100.0)$ & $(100.0)$ & $(100.0)$ & (100.0) & $(100.0)$ & & & \\
\hline
\end{tabular}

Color: $(0=$ dislike, $1=$ liked $)$; Aroma: $(0=$ dislike, $1=$ liked $)$; Cohesiveness: $(0=$ soft, $1=$ average, $2=$ hard $)$; Stickiness: $(0=$ not sticky, $1=$ average, $2=$ sticky); Taste: $(0=$ dislike, $1=$ liked $)$; Chewiness: $(0=$ dislike, $1=$ liked $)$; Texture: $(0=$ dislike, $1=$ liked).

\subsection{Ordered probit model results}

Separate models were estimated for couscous, fermented tuwo, and nonfermented tuwo using ordered probit. All explanatory variables are noncontinuous and entered as effect-coded variables (for example, taking the values of $[-1,1]$ rather than a series of $0-1$ dummy variables). The use of effect coding instead of dummy variables leads to marginal effect coefficients equal to partworths (Hamath et al., 1997). Results of the ordered probit models, major statistical properties, and specification test results are reported in Table 7. The interaction effects that were introduced to capture preference differences across ethnic groups are reported in Table 8. According to Maddala, the threshold coefficients or $\gamma_{i}$ 's should exhibit the following relationship $\gamma_{1} \leq \gamma_{2} \leq \cdots \leq \gamma_{w-2}$, and must be positive. Failure to exhibit any of these conditions would imply a specification error of the model. All threshold coefficients were positive and statistically significant at the 95\% confidence interval (Table 7) implying that there is no specification error.

A second test was done to look at the overall significance of the independent variables (levels of product attributes and consumer profile) in explaining the variation in the dependent variable rankings. A log-likelihood test distributed as $F_{551,5}$ with a critical value of 10.12 indicated strong rejection of the null hypothesis of the test, $v=b=0$ at the $95 \%$ confidence interval for all three food products. The $p$-values are 0 to 3 or more significant digits in all three cases. This means that the product attributes and consumer profile variables are relevant in explaining variation in consumer preferences. 
Table 7

Main effects estimates: Effects of product attributes on rankings and major statistical properties of the ordered probit models

\begin{tabular}{lccc}
\hline Product & & & \\
Variables & Fermented tuwo & Nonfermented tuwo & Couscous \\
\hline Constant & 0.250 & 0.239 & 0.691 \\
Color & 0.053 & $0.328^{* *}$ & $0.259^{* * *}$ \\
Aroma & -0.007 & -0.152 & 0.092 \\
Cohesiveness (hard at touch) & 0.109 & $0.171^{*}$ & $-0.156^{* * *}$ \\
Stickiness & $-0.283^{* *}$ & -0.008 & -0.090 \\
Taste & $0.335^{*}$ & $0.535^{* *}$ & $0.311^{* * *}$ \\
Chewiness & $0.313^{* *}$ & -0.042 & $0.310^{* * *}$ \\
Appearance (interior) & 0.096 & 0.258 & $0.252^{* *}$ \\
Consistency & $0.275^{*}$ & 0.119 & $0.790^{* * *}$ \\
Mouth feel & $0.169^{*}$ & & $1.432^{* * *}$ \\
Coefficient of the threshold variables & & $0.823^{* * *}$ & $2.214^{* * *}$ \\
$\quad \gamma_{1}$ & $0.754^{* * *}$ & $1.505^{* * *}$ & -760.941 \\
$\quad \gamma_{2}$ & $1.391^{* * *}$ & $2.213^{* * *}$ & -869.097 \\
$\quad \gamma_{3}$ & $2.098^{* * *}$ & -749.380 & 216.311 \\
Log-likelihood & -631.926 & -869.097 & 0.000 \\
Restricted log-likelihood (slopes $=0)$ & -716.200 & 239.433 & 551 \\
Chi-squared (18-18-12) & 168.548 & 0.000 & 551 \\
Significance level & 0.000 & & \\
Degree of freedom & 426 & & \\
\hline
\end{tabular}

${ }^{*}$ Denotes significance at the $10 \%$ level, ${ }^{* *}$ at the $5 \%$ level, and ${ }^{* * *}$ at the $1 \%$ level.

Lastly, the estimated coefficients listed in Table 7 were tested using a $t$-test. For nonfermented tuwo, color, cohesiveness, taste, consistency coefficients were found to be statistically significant at least at the $10 \%$ level. Stickiness, taste, chewiness, consistency, and mouth feel coefficients were significant at least at the $10 \%$ level for fermented tuwo. All couscous attributes except for aroma and stickiness were significant at the $1 \%$ level. The nonsignificance of a coefficient does not mean that the attribute or the level of the attribute is not important to consumers. Rather, it implies that consumers are indifferent to the proposed range of variation in the level of attributes.

\subsection{Partworth estimates}

The strength-of-choice experiment analysis is to decompose the revealed preference, i.e., rankings, of all individuals into marginal values or partworth estimates. Table 7 contains the main effect coefficients of product attributes that represent marginal values or partworths that a "typical" consumer, irrespective of background, places on attributes of couscous, fermented tuwo, or nonfermented tuwo. To illustrate, the color of a nonfermented tuwo product has a marginal value of 0.328 to an average consumer. Similarly, the taste of a fermented tuwo has a marginal value of 0.335 to an average consumer. Negative partworths mean that consumers' preference would decrease when attribute levels are increased. For example, for a typical fermented tuwo consumer, stickiness has a negative marginal value $(-0.283)$. Since utility or preference is an ordinal measure, the relative importance of those coefficients is more important than absolute magnitude.

Table 8 contains the estimated coefficients of interaction terms between the Haoussa ethnic group dummy variable and product characteristics. They represent the incremental value of preferences for products by members of the Haoussa ethnic group. For example, a typical Haoussa would value more the consistency of fermented tuwo, the cohesiveness of nonfermented tuwo, and the stickiness of couscous compared with the average Zarma consumer.

\subsection{Categorical classification tree results}

The categorical classification trees for the three final products are presented in Figs. 1-3. The variable that is being explained in the trees is the overall ranking of varieties, which ranges from 1 to 5 . Each node in the 
Table 8

Selected interaction effects of Haoussa ethnic dummy and product attributes

\begin{tabular}{lccc}
\hline Variables & $\begin{array}{l}\text { Fermented } \\
\text { tuwo }\end{array}$ & $\begin{array}{l}\text { Nonfermented } \\
\text { tuwo }\end{array}$ & Couscous \\
\hline Color & -0.237 & 0.128 & -0.231 \\
Aroma & -0.137 & -0.249 & 0.048 \\
Cohesiveness & 0.068 & $0.212^{*}$ & -0.015 \\
$\quad$ hard at touch) & & & \\
Stickiness & -0.058 & 0.022 & $0.331^{*}$ \\
Taste & 0.018 & 0.120 & -0.242 \\
Chewiness & 0.125 & -0.166 & 0.123 \\
Appearance & -0.041 & 0.05 & \\
Consistency & $0.274^{*}$ & 0.155 & \\
Mouth feel & 0.133 & 0.060 & \\
\hline
\end{tabular}

${ }^{*}$ Denotes significance at the $10 \%$ level, ${ }^{* *}$ at the $5 \%$ level, and $* * *$ at the $1 \%$ level.

trees contains the number of correct classifications of each preference value. For example, in the couscous classification tree in Fig. 1 the tree starts at the node with 114 observations on each of the preference values from 1 to 5 , representing the complete sample. Then when taste is used to split the sample and the taste is preferred (taste $=1$ ), the highest preference (5) is explained for 111 of the 114 observations. The branches of the tree are the attributes that are used to split the data. So, in the case of couscous taste, color, and chewiness explain the highest preference, and 106 out of 114 observations are correctly classified (bottom right in Fig. 1). This result is consistent with the ordered probit results that taste, color, and chewiness have the significant positive marginal values.

Fig. 2 presents the fermented tuwo classification tree. This figure reveals that taste, aroma, and consistency explain the highest preference for millet varieties. In this case the classification tree analysis adds significant information to the probit results because there is one significant difference between the classification tree results and the ordered probit results. The aroma of fermented tuwo has a statistically insignificant negative marginal value in the ordered probit analysis, but it correctly classifies 84 out of 89 of the highest preferences. The insignificant ordered probit coefficient is likely caused by inadequate variation in aroma preferences across the five levels of preference variety. In other words, most respondents found the aroma of fermented tuwo from the bottom four varieties to be about the same. Nevertheless, the aroma of

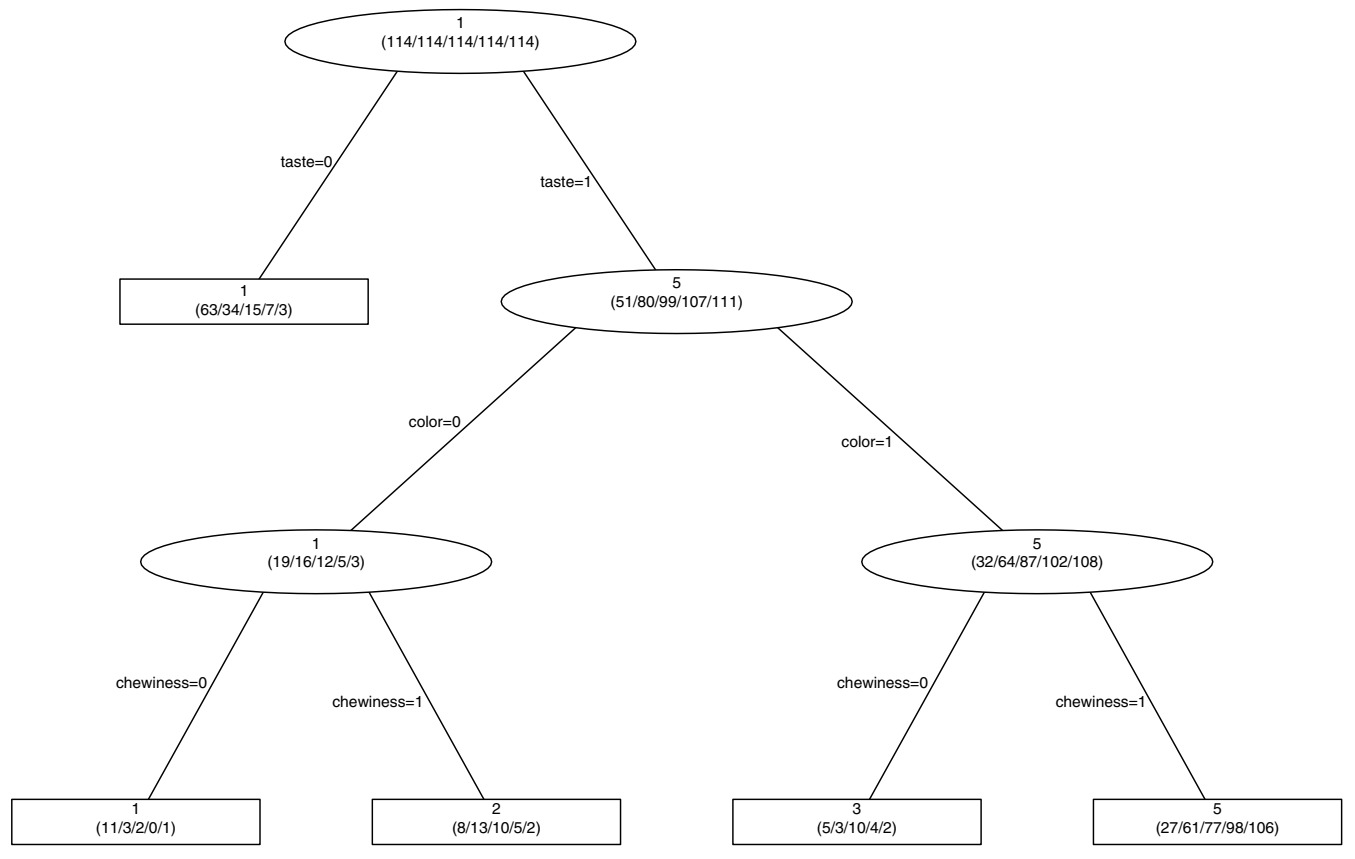

Fig. 1. Couscous classification tree. 


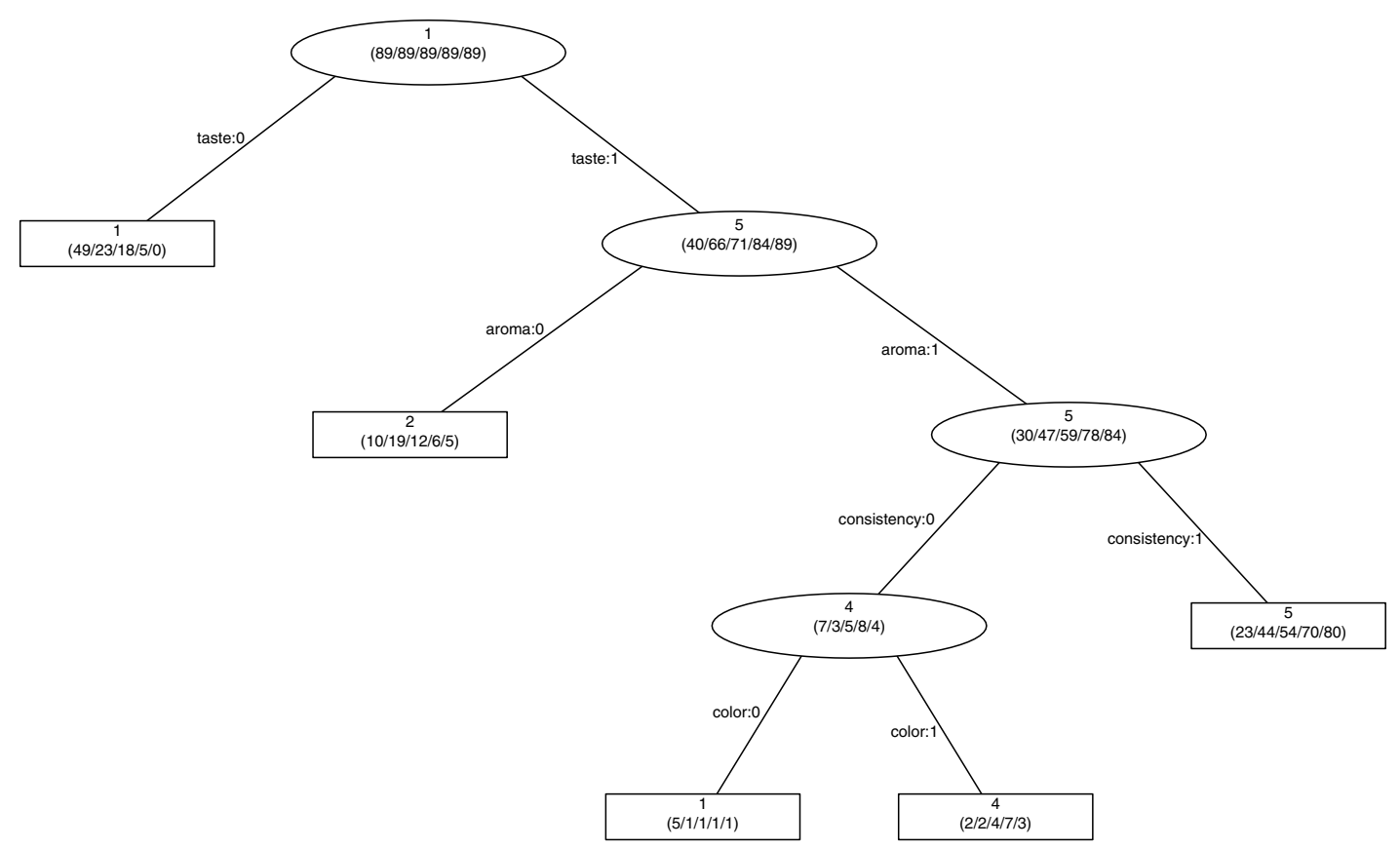

Fig. 2. Fermented towu classification tree.

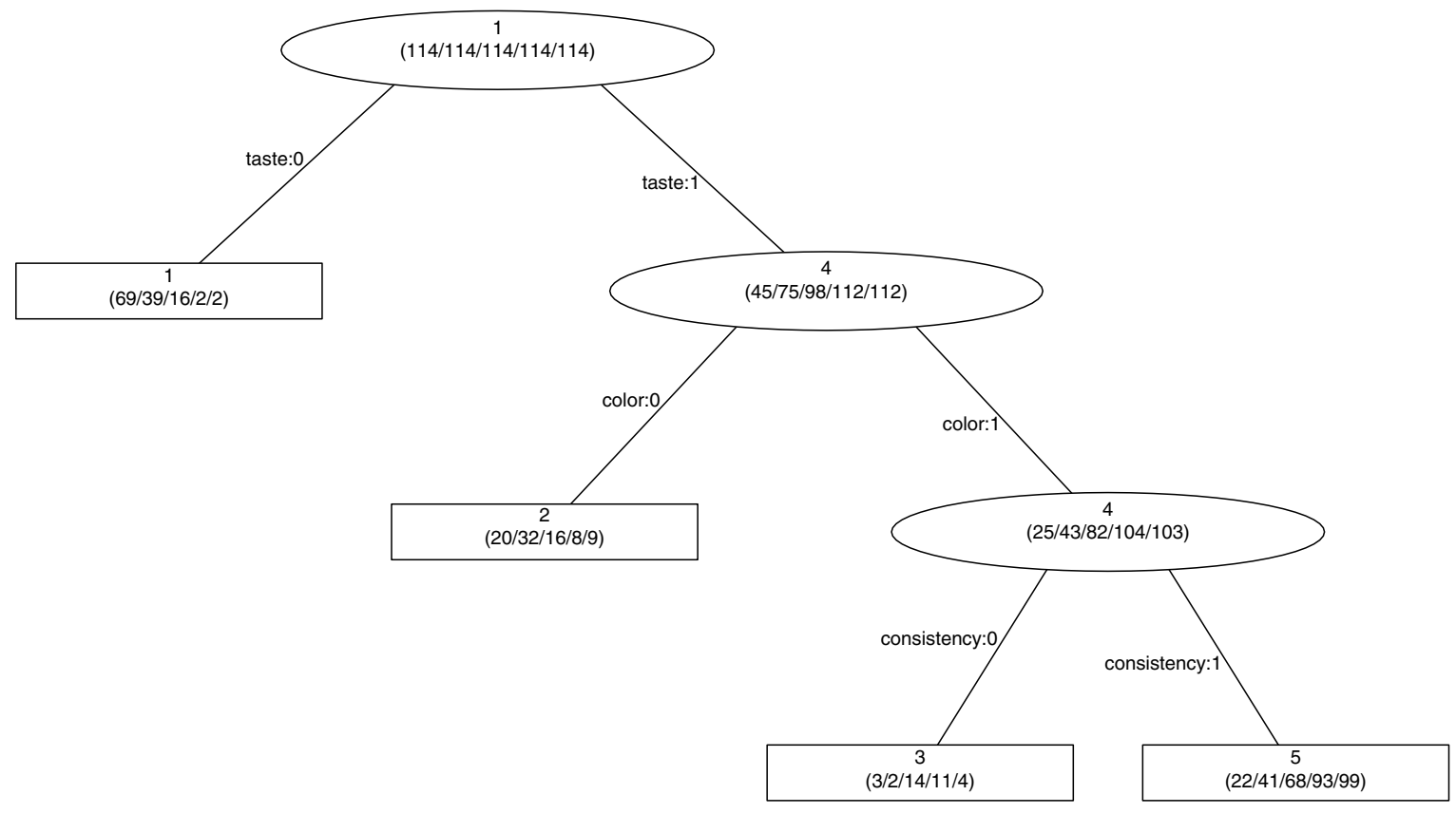

Fig. 3. Towu classification tree. 
the most preferred variety clearly distinguishes that variety from the other four. In addition, chewiness, which has a statistically significant marginal value of 0.313 , does not appear in the classification tree. This means that chewiness does not do a good job of discriminating between highest preference and lowest preference.

The classification tree results for tuwo, presented in Fig. 3, are very consistent with the ordered probit results. Taste, color, and consistency explain the highest preference for tuwo, with 99 out of 114 observations being correctly classified. These are the three variables with large, positive, and statistically significant coefficients in the ordered probit model. Thus these three attributes have large marginal values in tuwo consumption and explain the highest variety preference.

\subsection{Attribute value and relative importance of product attributes}

Partworth values are commonly utilized in choice experiment analysis. A large partworth value associated with an attribute level indicates high preferences for that particular level. These results are consistent with prior sorghum sensory studies. The criteria for a good sorghum tuwo found in Mali were the taste, color, texture, and keeping quality (Scheuring et al., 1982). In Burkina Faso and Mali, a good tuwo is one with a firm paste that holds together and does not crumble under finger pressure. Firmness and nonstickiness must remain constant when tuwo is stored overnight before consumption. Yellow or white tuwo is preferred, while pink, red, or gray tuwo may be rejected (Da et al., 1982). Similarly, in East and Southern Africa, desirable texture and keeping quality of light-colored ugali are the most important traits (Mukuru et al., 1982). Light-colored, white-cream, or yellowish-colored with good keeping quality are preferred by tuwo consumers in Nigeria. In Niger, stickiness of the tuwo in the mouth and cohesiveness were the most important traits. Taste and aroma were also proven to be important. Color, taste, and texture were found to be the most important traits preferred by couscous consumers in Niger (Oumarou et al., 1998).

Findings from this study indicate that taste, chewiness, color, and cohesiveness are the most important attributes preferred by couscous consumers. In effect, light, white creamed, or yellowish colors were found to be the most preferred color. These colors are similar to couscous, made of wheat, found in the market. Dark colors were found to be the least preferred. For nonfermented tuwo consumers, color, cohesiveness, taste, and consistency were the most important traits. Finally, stickiness, taste, chewiness, consistency, and mouth feel were found to be the most important fermented tuwo traits. Color was not found to be an important trait for fermented tuwo. In effect, fermentation may improve tuwo color if grains are soaked in an acid environment, as the $\mathrm{pH}$ level plays an important role in determining color. Acid tuwo color is always lighter than alkali tuwo (Scheuring et al., 1982). In this study, fermentation of grains may have improved the color of the tuwo. Thus, consumers may have unable to discern the color of the fermented tuwo. Keeping quality was found to be an important trait in many studies. However, in this study, significant and positive association were found between the ranking before and after overnight suggesting that for the varieties tested, keeping quality was consistent across varieties. In effect, varieties that were poorly rated the first day were also poorly rated the next. In effect, every time a tuwo has poor texture, it also has poor keeping quality. No exception was found to that rule (Scheuring et al., 1982).

In order of increasing importance, taste, color, texture, and keeping quality were the most important traits (Scheuring et al. 1982). Similarly, stickiness and cohesiveness (which are textural traits), taste, and aroma were also found to be the most important traits by order of importance in Niger (Aboubakar, 1992). The relative importance of product traits differs in this study. In effect, for nonfermented tuwo consumers; taste, color, consistency, and cohesiveness are the important traits by order of importance. The discrepancies from prior evaluation studies may be explained by the failure to account for multiple correlations that exist between traits. Most previous studies used simple correlation or single regression models to derive the relative importance of traits to consumers.

Table 9 shows the relative importance of traits for the three products to consumers. It is computed by taking the difference between the highest and the lowest partworth value of an attribute over the sum of the ranges for all attributes. Relative importance allows an attribute-to-attribute comparison. It indicates which attributes produce more value. For couscous, the taste and chewiness are the most important attributes followed by color and cohesiveness. For nonfermented 
Table 9

Relative importance of product attributes (\%)

\begin{tabular}{lccr}
\hline Product attributes & $\begin{array}{l}\text { Fermented } \\
\text { tuwo }\end{array}$ & $\begin{array}{l}\text { Nonfermented } \\
\text { tuwo }\end{array}$ & Couscous \\
\hline Color & 0 & 26 & 25 \\
Aroma & 0 & 0 & 0 \\
Cohesiveness & 0 & 13 & 15 \\
$\quad$ (hard at touch) & & & 0 \\
Stickiness & 21 & 0 & 30 \\
Taste & 24 & 42 & 30 \\
Chewiness & 23 & 0 & \\
Appearance (interior) & 0 & 0 & 100 \\
Consistency & 20 & 20 & \\
Mouth feel & 12 & 0 & \\
Total & 100 & 100 & \\
\hline
\end{tabular}

tuwo, taste, color, consistency, and cohesiveness are of decreasing importance. Finally, for fermented tuwo; taste, chewiness, stickiness, and mouth feel are important in decreasing order (Table 9). The results for couscous and nonfermented tuwo are consistent with the classification tree results, providing strong evidence of the importance of the identified attributes. The results for fermented tuwo are not as clear. Again, this may be because the fermentation process masks significant differences between varieties.

Combining these results, a consumption-oriented explanation of preferences for millet varieties would concentrate on the taste, color, and chewiness of couscous and the taste, color, and consistency of tuwo. These results highlight the importance of good taste and color attributes for household acceptance of millet varieties. Failure to produce light-colored couscous and tuwo with a taste like that of traditional varieties used in the household would result in household resistance to the adoption of new millet varieties.

\section{Summary and conclusion}

In order to develop varieties that are acceptable to producers and food processors, breeders must select for desirable traits. The primary objective of this study was to evaluate consumer preference for couscous and tuwo traits. Choice experiment analysis was used to estimate the marginal value of product attributes. Classification trees were used to identify the attributes that lead to the most preferred variety.
The most important attribute explaining preference is taste. This attribute is difficult to quantify. It refers to having the taste qualities that households prefer. These preferences are reflected in the consumption choices of the household. So, we can conclude that in order to make new varieties acceptable they must be bred to have a taste that is close to the taste of traditional varieties used in the household. To aid this breeding work food chemical analysis of varieties with preferred taste qualities are needed. The second most important attribute is color. Households want couscous and tuwo to have a light, creamy color. Absence of this color attribute will cause a new variety to be rejected by households. Of lesser importance in determining household acceptance are the chewiness of couscous and the consistency of tuwo. These taste preferences will play a significant role in household acceptance of new millet varieties and consumer acceptance of processed products from millet. Research is needed to correlate this consumer preference with measurable physical and chemical characteristics of alternative millet varieties.

\section{Acknowledgments}

The support of the International Sorghum and Millet Collaborative Research and Support Program (INTSORMIL), the International Crop Research Institute for the Semi-Arid Tropics (ICRISAT), and Institut National de la Recherche Agronomique du Niger (INRAN) is gratefully acknowledged.

\section{References}

Aboubakar, A., "Relationships between Consumer Ratings and Laboratory Measurements of Grain Sorghum Tuwo Parameters," Master's thesis, Purdue University (1992).

Agresti, A., Categorical Data Analysis (Wiley: New York, 1990).

Baidu-Forson, J., "On-Station Farmer Participatory Variety Evaluation: A Strategy for Client Oriented Breeding," Experimental Agriculture 33 (1997), 43-50.

Baidu-Forson, J., F. Waliyar, and B. Ntare, "Farmers Preferences for Socioeconomic and Technical Interventions in Groundnut Production System in Niger: Conjoint and Ordered Probit Analyses," Agricultural Systems 54 (1997), 463-476.

Boling, M., and N. Eisener, "Bogobe: Sorghum Porridge of Botswana," in Proceeding of an International Symposium of Sorghum Grain Quality, Patancheru, India (1982), pp. 32-35.

Breiman, L., J. Friedman, R. Olshen, and C. Stone, Classification and Regression Trees (Wadsworth Inc: Belmont, CA, 1984). 
Da, S., O. Akingbala, L. Rooney, J. Schuering, and F. Miller, "Evaluation to Tô Quality in a Sorghum Breeding Program," in Proceeding of an International Symposium on Sorghum Grain Quality (1982), pp. 11-23.

Doyle, P., "The Application of Probit, Logit, and Tobit in Marketing: Review," Journal of Business Research 5 (1977), 235-248.

Fliedel, G., C. Sambumukama, L. Songre, and F. Matencio, "Tests d'Évaluation de la Qualité des Grains de Sorgho Pour des Bouillies Épaisses Traditionnelles en Afrique," in l'Atelier Régional sur les semences et hybrides de Sorgho et de Mil (INRAN, Niamey, Niger, 1998).

Gebrekian, B., and B. GebreHiwot, "Sorghum Injera Preparations and Quality Parameters," in Proceeding of an International Symposium on Sorghum Grain Quality, Patancheru, India (1982), pp. 55-66.

Green, P., and D. DeSarbo, "Componential Segmentation in the Analysis of Consumer Tradeoffs," Journal of Marketing 43 (1979), 83-91.

Greene, W., Econometric Analysis (Macmillan: New York, 1990).

Hamath, A. S., M. D. Faminow, G. V. Johnson, and G. Crow, "Estimating the Values of Cattle Characteristics using an Ordered Probit Model," American Journal of Agricultural Economics 79 (1997), 463-476.

INRAN, "Catalogue de Varietés Développées au Niger. Technical Report, Institut de la Recherche Agronomique du Niger," Ministére de l'Agriculture et de l'Elevage (1984).

Jain, A., F. Acito, N. Malhotra, and V. Mahajan, "A Comparison of Internal Validity of Alternative Parameter Estimation Methods in Decompositional Multiattribute Preference Models," Journal of Marketing Research 16 (1979), 313-322.

Jeannette, M., M. Oumarou, A. Kirleis, and J. Clark, "Manuel de Laboratoire Pour Analyses de la Qualité du Sorgho Pour Usage dans l' Afrique de l'Ouest," Technical report, Institut Naitonal de la Recherche Agronomique (INRAN) (1987).

Lancaster, K., Modern Consumer Theory (Edward Elgar: Aldershot, UK, 1991).

Louviere, J., Analysing Decision Making: Metric Conjoint Analysis. Quantitative Applications in Social Sciences (Sage: Beverly Hills, CA, 1990).

Louviere, J., "Choice Experiments: An Overview of Concepts and Issues," in J. Bennett and R. Blamey (Eds.), The Choice Modeling Approach to Environmental Evaluation (Edward Elgar: Northampton, MA, 2001), pp. 13-36.

Maddala, G., Limited-Dependent and Qualitative Variables in Econometrics (Cambridge University Press: Cambridge, UK, 1993).

Moore, W., "Levels of Aggregation in Conjoint Analysis: An Empirical Comparison," Journal of Marketing Research 17 (1980), 516-523.

Mukuru, S., J. Mushonga, and D. Murty, "Sorghum Ugali," in Proceeding of an International Symposium on Sorghum Grain Quality, Patancheru, India (1982), pp. 31-44.
Murty, D., and K. Kumar, "Traditional Uses of Sorghum and Millets," in D. Dendy (Ed.), Sorghum and Millets: Chemistry and Technology (Ciba-Geigy: Greensboro, NC, 1995), pp. 185221.

Murty, D., H. Patil, and L. House, "Sankati Quality Evaluation of Sorghum Cultivars," in Proceeding of an International Symposium on Sorghum Grain Quality, Patancheru, India (1982), pp. 36-38.

Ndjeunga, J., "Constraints to Variety Release, Seed Multiplication and Distribution of Sorghum, Pearl Millet and Groundnut in West and Central Africa," in Alternative Strategies for Smallholder and Seed Supply. Proceedings of an International Conference on Options for Strengthening National and Regional Seed Systems in Africa and West Asia, Harare, Zimbabwe. International Crop Research Institute for the Semi-Arid Tropics (1997), pp. 3446.

Nelson, C., and J. Ndjeunga, "Prospects for a Pearl Millet and Sorghum Food Processing Industry in West Africa," in Proceedings of a Regional Workshop Organized by the West and Central Africa Sorghum Research Network (WCASRM), Lome, Togo (1999).

Obilana, A., "Traditional Sorghum Foods in Nigeria: Their Preparation and Quality Parameters," in Proceeding of an International Symposium on Sorghum Grain Quality, Patancheru, India (1982), pp. 45-54.

Oumarou, M., S. Kaka, and M. Moustapha, "Caractérisation Physique, Chimique et Nutritionnelle des Varietés Améliorées de Mil," Technical report, Institut National de la Recherche Agronomique du Niger (INRAN). Laboratoire de Technologie Alimentaire (1998).

Ratchford, B., "The New Economic Theory of Consumer Behavior: An Interpretive Essay," Journal of Consumer Research 2 (1975), $65-75$.

Robins, E., "Evaluation des Essais en Milieu Réel et de l'etat des Ressources Naturelles par les Agriculteurs. Resultats des Enquêtes d'Opinions Auprès des Agriculteurs 1990-94. Zone Centrale, Burkina Faso," in Recherche Intregrée en Production Agricole et en Gestion des Ressources Naturelles: Projet d'appui à la Recherche et à la la Formation Agricole (ARTS) (Purdue University and Winrock International: West Lafayette, IN, 1995).

ROCAFREMI, "Enquête sur les Produits du Mil en Afrique de l'Ouest" (1997).

Scheuring, J., S. Sidibe, and A. Kante, "Sorghum Alkali Towu Quality Considerations," in Proceedings of an International Symposium on Sorghum Grain Quality, Patancheru, India (1982, October), pp. 21-31.

Train, K., Qualitative Choice Analysis: Theory, Econometrics, and an Application to Automobile Demand (MIT Press: Cambridge, MA, 1986)

Venables, W., and B. Ripley, Modern Applied Statistics with S-PLUS, 3d ed (Springer: New York, 1999). 